\title{
Article \\ Characteristics of Biodegradable Gelatin Methacrylate Hydrogel Designed to Improve Osteoinduction and Effect of Additional Binding of Tannic Acid on Hydrogel
}

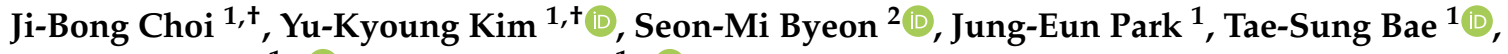 \\ Yong-Seok Jang ${ }^{1, *(\mathbb{D})}$ and Min-Ho Lee ${ }^{1, *(\mathbb{D})}$ \\ 1 Department of Dental Biomaterials, Institute of Biodegradable Materials, School of Dentistry, \\ Jeonbuk National University, Jeonju-si 54896, Jeollabuk-do, Korea; submissi@naver.com (J.-B.C.); \\ yk0830@naver.com (Y.-K.K.); pje312@naver.com (J.-E.P.); bts@jbnu.ac.kr (T.-S.B.) \\ 2 Dental Clinic of Ebarun, Suncheon-si 57999, Jeollanam-do, Korea; sumse1205@naver.com \\ * Correspondence: Yjang@jbnu.ac.kr (Y.-S.J.); mh@jbnu.ac.kr (M.-H.L.) \\ + These authors contributed equally to the paper.
}

Citation: Choi, J.-B.; Kim, Y.-K.; Byeon, S.-M.; Park, J.-E.; Bae, T.-S.; Jang, Y.-S.; Lee, M.-H. Characteristics of Biodegradable Gelatin Methacrylate Hydrogel Designed to Improve Osteoinduction and Effect of Additional Binding of Tannic Acid on Hydrogel. Polymers 2021, 13, 2535. https://doi.org/10.3390/ polym 13152535

Academic Editors: Vetcher Alexandre and Alexey Iordanskii

Received: 16 July 2021

Accepted: 28 July 2021

Published: 31 July 2021

Publisher's Note: MDPI stays neutral with regard to jurisdictional claims in published maps and institutional affiliations.

Copyright: (c) 2021 by the authors. Licensee MDPI, Basel, Switzerland. This article is an open access article distributed under the terms and conditions of the Creative Commons Attribution (CC BY) license (https:// creativecommons.org/licenses/by/ $4.0 /)$.

\begin{abstract}
In this study, a hydrogel using single and double crosslinking was prepared using GelMA, a natural polymer, and the effect was evaluated when the double crosslinked hydrogel and tannic acid were treated. The resulting hydrogel was subjected to physicochemical property evaluation, biocompatibility evaluation, and animal testing. The free radicals generated through APS/TEMED have a scaffold form with a porous structure in the hydrogel, and have a more stable structure through photo crosslinking. The double crosslinked hydrogel had improved mechanical strength and better results in cell compatibility tests than the single crosslinked group. Moreover, in the hydrogel transplanted into the femur of a rat, the double crosslinked group showed an osteoinductive response due to the attachment of bone minerals after 4 and 8 weeks, but the single crosslinked group did not show an osteoinductive response due to rapid degradation. Treatment with a high concentration of tannic acid showed significantly improved mechanical strength through H-bonding. However, cell adhesion and proliferation were limited compared to the untreated group due to the limitation of water absorption capacity, and no osteoinduction reaction was observed. As a result, it was confirmed that the treatment of high-concentration tannic acid significantly improved mechanical strength, but it was not a suitable method for improving bone induction due to the limitation of water absorption.
\end{abstract}

Keywords: gelatin methacryloyl; osteoinduction; tannic acid; crosslinking; hydrogel; biodegradable

\section{Introduction}

Bone defects are health-threatening diseases and are caused by various factors such as trauma, genetics, and cancer. The number of patients increases with age. Although bones can be regenerated, the ability widely varies from person to person. Currently, the most common method to recover the damaged bone defects is the direct implantation of a bone-grafted material into the defective area. Bone grafting must include essential elements of bone regeneration, namely osteoinduction, osteoconduction, and osteogenesis, in conjunction with the final bonding between the bone and the graft material [1]. In bone tissue engineering, various complex processes involving cell adhesion, migration, proliferation, differentiation, and matrix formation are used while applying biomaterials to induce bone generation [2]. To recover functions, often, biomaterials containing bioactive substances are used [3]. Hydrogels made of natural and synthetic biomaterials that similarly mimic the structure and biological properties of the natural extracellular matrix have long been studied as candidates for cell delivery in medicine and dentistry [4].

Gelatin is a type of derived protein partially extracted from collagen by thermal or chemical denaturation. It is suitable for hydrogels due to its retentive ability for a 
motif of peptides that are degraded by matrix metalloproteinase (MMP) and arginineglycine-aspartic acid (RGD) related to cell adhesion. Additionally, gelatin has an excellent biocompatibility and swelling ratio [5-8]. However, gelatin dissolves in water at a body temperature above $37^{\circ} \mathrm{C}$ and does not offer structural stability. Its physical properties are improved by chemical crosslinking with glutaraldehyde or 1-ethyl-3-(3dimethylaminopropyl)-carbodiimide) (EDC)/(N-hydroxysuccinimide) (NHS) [9,10]. However due to crosslinker toxicity, many restrictions were imposed as referred from previous studies [11-13].

The recently developed gelatin methacryloyl (GelMA) can be produced by chemical modification of the amine and hydroxyl groups of gelatins, through which the hydrogel can be covalently crosslinked in the presence of photoinitiators and light [14]. In addition, GelMA hydrogels irreversibly change some structures due to hydrolysis and chemical modification but retain some properties of collagen and gelatin, such as cell adhesion, heat sensitivity, and enzymatic degradation [6,14,15]. GelMA hydrogels support the formation of novel ECMs, are enzymatically degradable, can be produced at low cost, are readily crosslinked under physiological conditions, and show potential for tissue engineering [16].

Irgacure 2959 (2-hydroxy-4'-(2-hydroxyethoxy)-2-methylpropiophenone) is the most commonly used in tissue engineering applications [17-20]. However, its low water solubility and UV light ( $365 \mathrm{~nm}$ ) exposure cause potentially harmful effects on cells and tissues. Prolonged exposure to UV light may damage the DNA and cellular functions [21-23]. However, visible light (VL) uses a longer wavelength $(405 \mathrm{~nm})$ and penetrates further into the tissue during treatments. Additionally, no heat is generated, and cell damage is minimal [24]. The biocompatibility of GelMA hydrogel in bone tissue engineering has been demonstrated by many studies [25-30]. However, hydrogels fabricated with GelMA have lower mechanical strength than other natural and synthetic polymeric hydrogels [31-33].

Recently, hydrogels made with double crosslinking (DC) are attracting a lot of attention due to their excellent mechanical performance [34]. In addition, methods have been proposed to adjust the mechanical properties of GelMA using several crosslinking steps. For example, Rizwan et. al. achieved double crosslinking by performing physical crosslinking and then photo crosslinking [35]. In another study, Zhou et. al. used enzyme crosslinking followed by photo crosslinking to improve the viscosity of GelMA for bioprinting [36].

Tannic acid (TA) is a natural polyphenol compound with biological antioxidant and antibiotic properties [37]. However, TA forms an amorphous structure with a complex coagulation behavior in hydrogels making it difficult to control these strong interactions $[38,39]$. Accordingly, macromolecules such as DNA proteins are used to balance the change [40], or multiple steps are applied on TA under controlled conditions [41]. Similar to polydopamine inspired by mussels, a high pyrogallol and catechol content of the TA molecular structure can improve compressive and tensile properties via bonding [42].

In this study, based on the excellent binding ability of the GelMA hydrogel fabricated using double crosslinking and TA (see Figure 1), it was evaluated through analysis whether the hydrogel network could be strengthened in a well-arranged manner by TA. In addition, changes in mechanical properties were observed when the double crosslinked hydrogel and TA were applied to the hydrogel, and the effect on biodegradation and osteoinduction when finally implanted in an animal model was observed. Based on this, it was attempted to confirm whether the manufacturing method using double crosslinking showed better effects than the manufacturing method using single crosslinking. In addition, we tried to determine whether the improvement of mechanical strength when TA was combined and whether TA had an effect on the improvement of bone induction. 


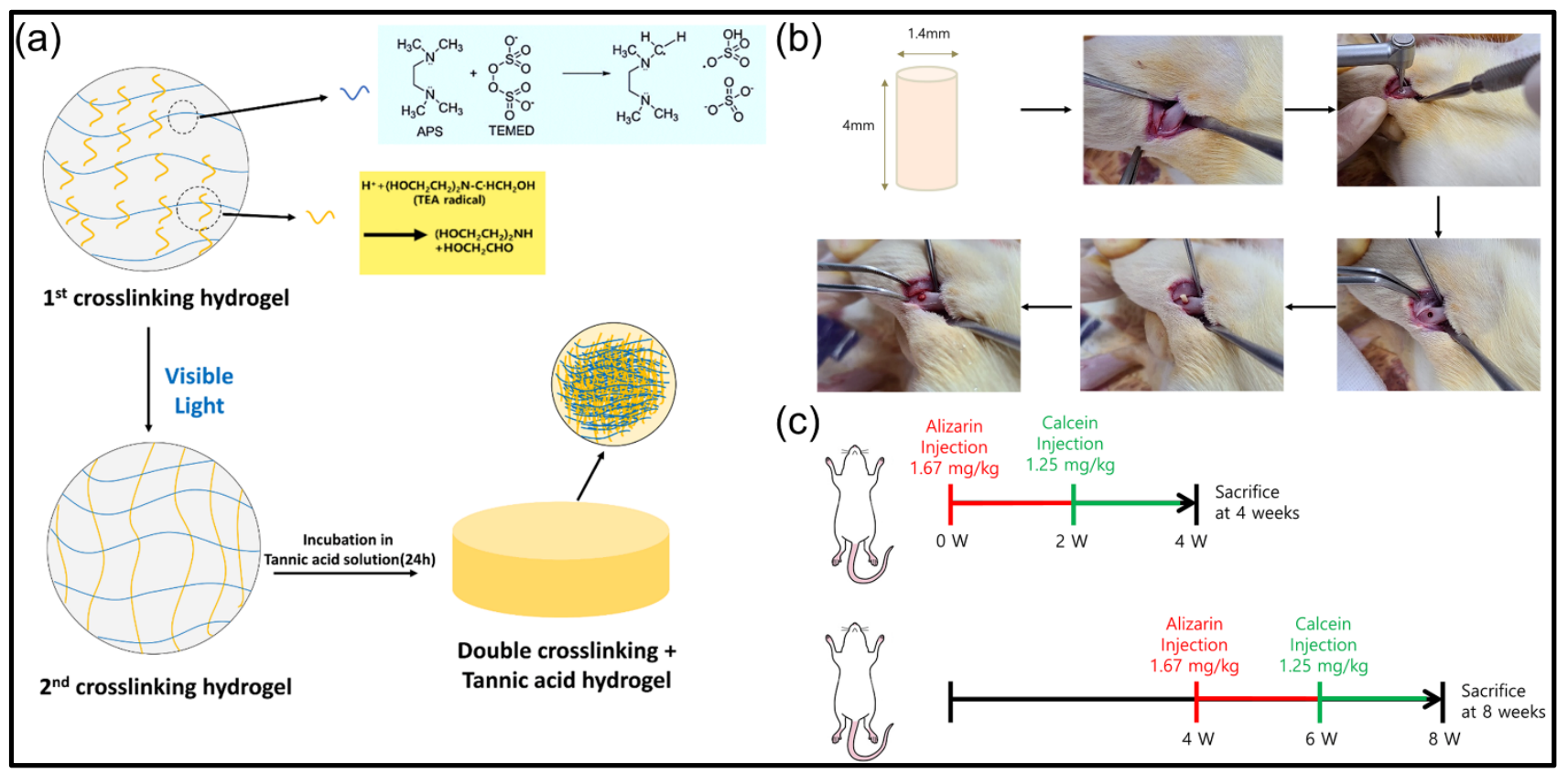

Figure 1. Schematic diagram of (a) Fabrication of double crosslinked hydrogel and tannic acid treatment, (b) Sample type and location for in vivo test (Sample placement in the femur), (c) Group division according to the schedule of staining reagent injection time for checking and analyzing bone formation behavior.

\section{Materials and Methods}

All materials used in this experiment, including type A gelatin, tetramethyl ethylene diamine (TEMED), ammonium persulfate (APS), methacrylic anhydride (MA), and tannic acid (TA), were purchased from Sigma Aldrich (Yongin, Korea). All chemicals were used without further purification.

\subsection{Systhesis of Gelatin Methacryloyl}

According to a previously reported study [43], methacryloyl-bonded GelMA macromonomer was synthesized. A total of $10 \%(w / v)$ of gelatin was completely dissolved in Dulbecco's phosphate-buffered saline (DPBS) at $60^{\circ} \mathrm{C}$ and magnetically stirred. Then, $8 \mathrm{~mL}$ of MA were added to the gelatin solution and stirred at $50{ }^{\circ} \mathrm{C}$ for $2 \mathrm{~h}$. The GelMA solution was then diluted in pre-made DPBS to increase the volume by 5 times and terminate the reaction. The GelMA solution was dialyzed against deionized water for 1 week at $40{ }^{\circ} \mathrm{C}$ in a 12-14 kDA cutoff tube. Subsequently, the solution was freeze-dried for 5 days, and the resulting GelMA was stored in a $-20^{\circ} \mathrm{C}$ freezer until further use.

\subsection{Fabrication of Single and Double Crosslinking Hydrogels}

The concentration of GelMA solution was fixed at 15\% based on previous experimental results [44]. The prepared GelMA foam was dissolved in deionized water at $50{ }^{\circ} \mathrm{C}$ for $2 \mathrm{~h}$. The hydrogel prepared by single crosslinking was prepared using low-temperature crosslinking and light crosslinking.

A hydrogel using low-temperature crosslinking was prepared by dissolving $14 \%(w / v)$ ammonium persulfate (APS) and $7 \%(w / v)$ tetramethylethylenediamine (TEMED). This prepolymer solution was pipetted into cylindrical ( $1.5 \mathrm{~mm}$ diameter, $1 \mathrm{~mm}$ thickness) polystyrene molds and placed in a freezer set to $-20{ }^{\circ} \mathrm{C}$. Low-temperature crosslinking was allowed to proceed for $18 \mathrm{~h}$, and the resulting hydrogel was thawed and hydrated in $\mathrm{dH}_{2} \mathrm{O}$ prior to use.

For photo crosslinking, $1.88(v / v)$ triethanolamine (TEA), $1.25(w / v)$ Vinylcaprolactam (VC), and $0.5 \mathrm{mM}$ Eosin Y disodium salt were sequentially added to the prepared $15 \%$ $(w / t)$ GelMA solution and mixed, and then the prepolymer solution was pipetted into a 
cylindrical (1.5 mm diameter, $1 \mathrm{~mm}$ thick) polystyrene mold and exposed to visible light for $120 \mathrm{~s}$.

For double crosslinking, $14 \%(w / v)$ ammonium persulfate (APS) and $7 \%(w / v)$ tetramethylethylenediamine (TEMED) were sequentially added to the prepolymer solution used for photo crosslinking and mixed. Then, low-temperature crosslinking was per-formed in a freezer set at $-20^{\circ} \mathrm{C}$. for $18 \mathrm{~h}$, and then exposed to visible light for $120 \mathrm{~s}$ before thawing to form a double crosslinked hydrogel.

\subsection{Fabrication of Hydrogel Applied with TA}

The fabricated hydrogel was immersed in the tannic acid (TA) solution of previously pre-pared concentrations $(10 \%, 50 \%$, and $100 \%(w / v))$, and shaken for $24 \mathrm{~h}$ on a shaker. Then, the hydrogel was washed three times with deionized water to remove excess TA. Hydrogels used for the experiment were fabricated with a $10 \mathrm{~mm}$ diameter and a $3 \mathrm{~mm}$ height and were referred to as GelMA-S (low-temperature crosslinking), GelMA-V (photo crosslinking), and GelMA-D (double crosslinking). Depending on the concentration of TA, additional indicators, T10, T50, and T100, were used.

\subsection{Fourier Transform Infrared Spectroscopy (FT-IR) Characterization}

FT-IR analysis was used to investigate the intermolecular interactions between TA, double crosslinking, and the GelMA Spectra that were obtained at room temperature using a FT-IR spectrometer (Perkin Elmer, Waltham, MA, USA). FT-IR analysis was performed within the wavelength $4000-500 \mathrm{~cm}^{-1}(\mathrm{KBr})$ using the attenuated total reflectance (ATR) method.

\subsection{Mechanical Tests}

Mechanical properties of the hydrogel were measured using a universal testing machine (Instron 5569, Instron, Norwood, MA, USA). All the first compression tests were performed on a cylindrical hydrogel at a $2 \mathrm{~mm} / \mathrm{min}$ rate with up to $95 \%$ maximum load of a $50 \mathrm{~N}$ load cell. After the first compression test, damaged samples were eliminated, and the second compression test was conducted at a $0.5 \mathrm{~mm} / \mathrm{min}$ rate and up to a $95 \%$ maximum load of a $500 \mathrm{~N}$ load cell. The second compression test data were automatically calculated using the Bluehill 2 software. The compressive modulus was calculated as the slope of the linear region ( $0-20 \%)$ of the stress-strain curve. All samples were hydrated during the test.

\subsection{Swelling Ratio Tests}

The prepared GelMA hydrogel was incubated at $37^{\circ} \mathrm{C}$ for $24 \mathrm{~h}$. After the sample was removed from the solution and the residual liquid separated using Kimwipe, the weight, $\mathrm{W}_{\mathrm{s}}$, was measured. The weight of the freeze-dried hydrogel was measured as $\mathrm{W}_{\mathrm{d}}$. The swelling ratio was calculated according to Equation (1).

$$
\text { Swelling Ratio: } \mathrm{SR}=\left(\mathrm{W}_{\mathrm{s}}-\mathrm{W}_{\mathrm{d}}\right) / \mathrm{W}_{\mathrm{d}}
$$

\subsection{FE-SEM Characterization}

The freeze-dried hydrogel was placed on a wafer for platinum coating. The shape of the hydrogel was observed using a FE-SEM (Hitachi, Tokyo, Japan).

\subsection{Evaluation of In-Vitro Cell Proliferation}

Osteoblast cells were used in this study, MC3T3-E1 (ATCC; American Type Culture Collection), to evaluate their effect on bone formation. For the culture medium, a nutrient component, $10 \%$ fetal bovine serum (Gibco Co., Waltham, MA, USA) containing an antibiotic (penicillin), was added to an $\alpha$-MEM (Gibco Co., Waltham, MA, USA) medium. The cell culture was conducted in an incubator (Thermo Electron Corporation, Waltham, MA, USA) in a $5 \% \mathrm{CO}_{2}$ atmosphere at $37^{\circ} \mathrm{C}$. A water soluble tetrazolium (WST) assay was 
used to evaluate cell proliferation by placing samples in a 48-well plate and incubating the MC3T3-E1 cells with a cell density of $1 \times 10^{4}$ cells $\mathrm{mL}^{-1}$ for 1,3 , and 7 days. Then, the medium was removed, replaced with $400 \mu \mathrm{L}$ of CCK-8 (Enzo Life Science Inc., Farmingdale, NY, USA) reagent mixed with $\alpha$-MEM medium, and stored in the incubator with $5 \%$ of $\mathrm{CO}_{2}$. After $90 \mathrm{~min}, 100 \mu \mathrm{L}$ were added to a 96-well plate, and the absorbance was measured at $450 \mathrm{~nm}$ using the ELISA reader (Molecular devices, Silicon Valley, CA, USA).

\subsection{Evaluation of Bone Regeneration and Mineral Activity In Vivo}

The effect of bone remodeling was compared to the rat femur defect model applying GelMA-P, GelMA-PT100, GelMA-VP, and GelMA-VPT100 groups $(n=3)$. Experiments in this study were conducted under the protocols approved by the Institutional Animal Care and Use Committee of the Chonbuk National University Laboratory Animal Center (CBNU 2020-094) following the declaration of Helsinki. The prepared freeze-dried hydrogel samples were implanted on the proximal femur from the outer side for each rat. Male Sprague-Dawley rats $(n=16)$, used in this experiment, were about 8 weeks old with an average weight of $280 \mathrm{~g}$. The rats were purchased from Damul Science (Daejeon, Korea) and used after a week's adjusting period. For anesthesia, $0.6 \mathrm{~mL} / \mathrm{kg}$ tiletamine and zolazepam (Zoletil 50, Virbac Laboratories, Carros, France) and $0.4 \mathrm{~mL} / \mathrm{kg}$ xylazine hydrochloride (Rompun, Bayer Korea, Seoul, Korea) were intramuscularly injected into the leg of each rat. The surgical site of the anesthetized rat was shaved, and an approximately $1 \mathrm{~cm}$ incision was made on the femur after sterilization using povidone iodine. After raising the flap due to the incision, a contra-angle handpiece (X-smart Endodontic Motor, Dentsply Maillefer, Switzerland) equipped with a $1.6 \mathrm{~mm}$ pilot round head bur (H1.31-0.16, Lemgo, Germany) was used to create a hole in the cortical bone. After the surgery, an antibiotic (Amikacin, Samu Median Co., Ltd., Seoul, Korea) was subcutaneously injected $(0.6 \mathrm{~mL} / \mathrm{kg})$. At 4 and 8 weeks after implanting the hydrogels, the rats were sacrificed to obtain the femoral bone blocks containing the sample.

\section{Fluorescence Staining}

Alizarin complexone (red) and calcein (green) fluorescent materials were used to observe the mineralized bones. To evaluate a bone-forming ability on the samples after 4 and 8 weeks, Alizarin complexone (red) solution $(1.67 \mathrm{~mL} / \mathrm{kg}$, body weight) was injected at 0 and 4 weeks, and calcein $(1.25 \mathrm{~mL} / \mathrm{kg}$, body weight) was injected into the peritoneum at week 2 and week 6 . After 4 and 8 weeks, the femoral bone blocks were obtained from the sacrificed rats and were fixed in $10 \%$ formaldehyde solution to fabricate a resin-embedded tissue slide. Then, all blocks were dehydrated using an increased concentration of ethanol, and methyl methacrylate (MMA, JUSEI Chemical Co. Ltd., Tokyo, Japan) was inserted into the bone. The bone blocks with penetrated MMA were embedded in an activated MMA resin. The embedded blocks in the resin were sectioned along the longitudinal axis of the embedded sample. The fluorescent-stained tissue slide of the sectioned sample was observed using a Super Resolution Confocal Laser Scanning Microscope (Carl Zeiss AG, Oberkochen, Germany). Histological images of bone staining with alizarin complexone (red) and calcein (green) were obtained at $543 \mathrm{~nm}$ and $488 \mathrm{~nm}$, respectively.

\subsection{Statistical Processing}

To evaluate the difference among groups, SPSS ver 21.0 (SPSS Inc., Chicago, IL, USA) software was used. One-way ANOVA was used to assess three or more groups within one factor, and Tukey's postmortem analysis was used to evaluate the average. In all experiments, if the $p$-value was $<0.05$, it would determine significant differences in the groups. 


\section{Results and Discussion \\ 3.1. Formation of GelMA and TA Treatment of GelMA Hydrogel}

We prepared a hydrogel synthesized by single crosslinking and double crosslinking according to the plan, and TA solutions with different concentrations were applied. As shown in Figure 2a, it can be seen that GelMA-D has changed color under the influence of Eosin Y. In addition, GelMA (15\% w/v) hydrogel (cylindrical height $2 \mathrm{~mm}$, diameter $10 \mathrm{~mm})$ changed to opaque with a decrease in size after $24 \mathrm{~h}$ treatment of TA $(10 \%, 50 \%$, $100 \% w / v)$, which shows a real interaction between GelMA and TA.

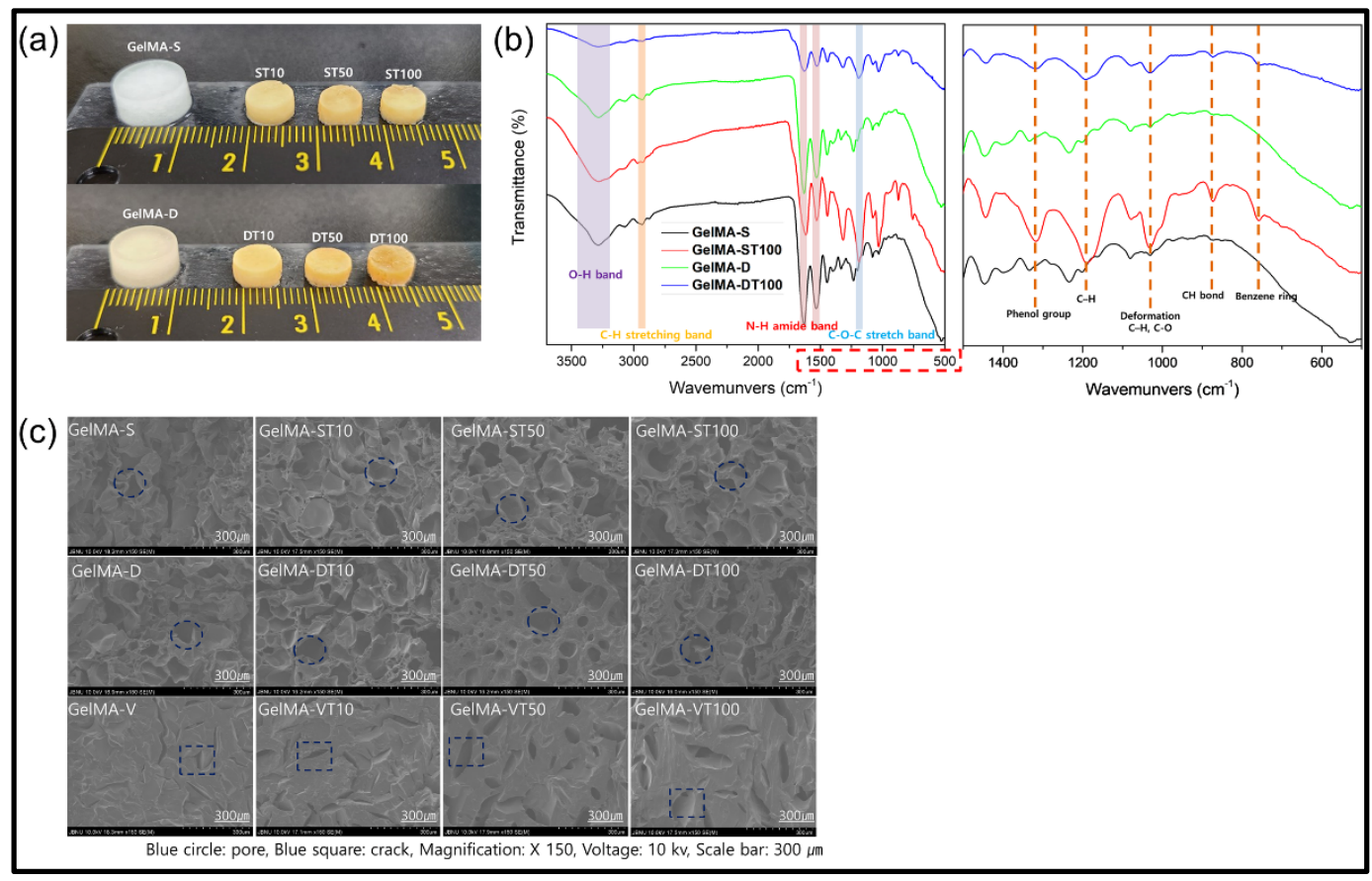

Figure 2. (a) The representative morphological changes from pristine GelMA hydrogels (left) to GelMA-TA (right) after a $24 \mathrm{~h}$ incubation in TA solution. Concentrations of GelMA and TA are 15\% (w/v), and 10\%, 50\%, 100\% (w/v), respectively. (b) FT-IR spectra of hydrogels. (c) FE-SEM of GelMA hydrogels, and GelMA-TA hydrogel 10\%, 50\%, 100\% TA, $24 \mathrm{~h}$ treatment time. Red circles indicate pores, and squares indicate cracks. The image was measured using a voltage of $10 \mathrm{kv}$, the magnification was $150 \times$, and the scale bar was $300 \mu \mathrm{m}$.

To confirm the interaction further, the Fourier Transform Infrared (FT-IR) study was performed (Figure 2b). Spectra from the remaining groups except for GelMA-S generally show that high transmittance at about $3100-3600 \mathrm{~cm}^{-1}$ is closely related to hydrogen bonding, which is due to the shift of $\mathrm{O}-\mathrm{H}$ groups by additional bonding [45,46]. In addition, the peaks of the amide groups (I, II, III) characteristic of GelMA hydrogels were observed at around $1612 \mathrm{~cm}^{-1}, 1520 \mathrm{~cm}^{-1}, 1436 \mathrm{~cm}^{-1}$ without appreciable changes in intensity and frequency. These results show that covalent bonds are not present [47]. The peak at $1319 \mathrm{~cm}^{-1}$ is caused by the phenol group of TA. The peak at $1198 \mathrm{~cm}^{-1}$ is due to C-H, and the vibration peak at $1100-1000 \mathrm{~cm}^{-1}$ is due to C-O and C-H deformation. The peak in $550-900 \mathrm{~cm}^{-1}$, which is characteristic of TA, is based on the C-H bond of the benzene ring [48].

Morphological analysis of the prepared GelMA and TA treatment of GelMA hydrogels was measured by FE-SEM (Figure 2c). For comparison, a hydrogel (GelMA-V) formed by photo crosslinking was additionally observed. GelMA-V showed agglomerated surfaces and partial cracks due to polymerization by radicals. The hydrogel formed by low-temperature crosslinking (GelMA-S) had a porous microstructure as the ice crystals formed by the APS/TEMED reaction were removed. The hydrogel formed by double crosslinking (GelMA-D) was photo crosslinked after low-temperature crosslinking and 
showed a similar shape to GelMA-S. Although an increase in pore size was observed with increasing TA concentration $(10 \%, 50 \%$, and $100 \%)$ in GelMA-V, the size did not increase noticeably after $50 \%$ concentration. In GelMA-S, it was confirmed that the wall of the porous structure was slightly thickened by the binding of TA, and in GelMA-D, more TA binding than GelMA-P was observed. However, looking at the overall trend, no significant difference in surface shape was observed between GelMA-S and GelMA-D groups.

\subsection{Mechanical Properties of Htdrogel}

Figure 3a shows a typical compressive stress-strain curve. In the case of the TA-treated group, it was confirmed that the fracture stress was significantly improved. In particular, the untreated and TA-treated hydrogel groups showed the same pattern and showed stronger fracture stress in the double crosslinked group than in the single crosslinked group.

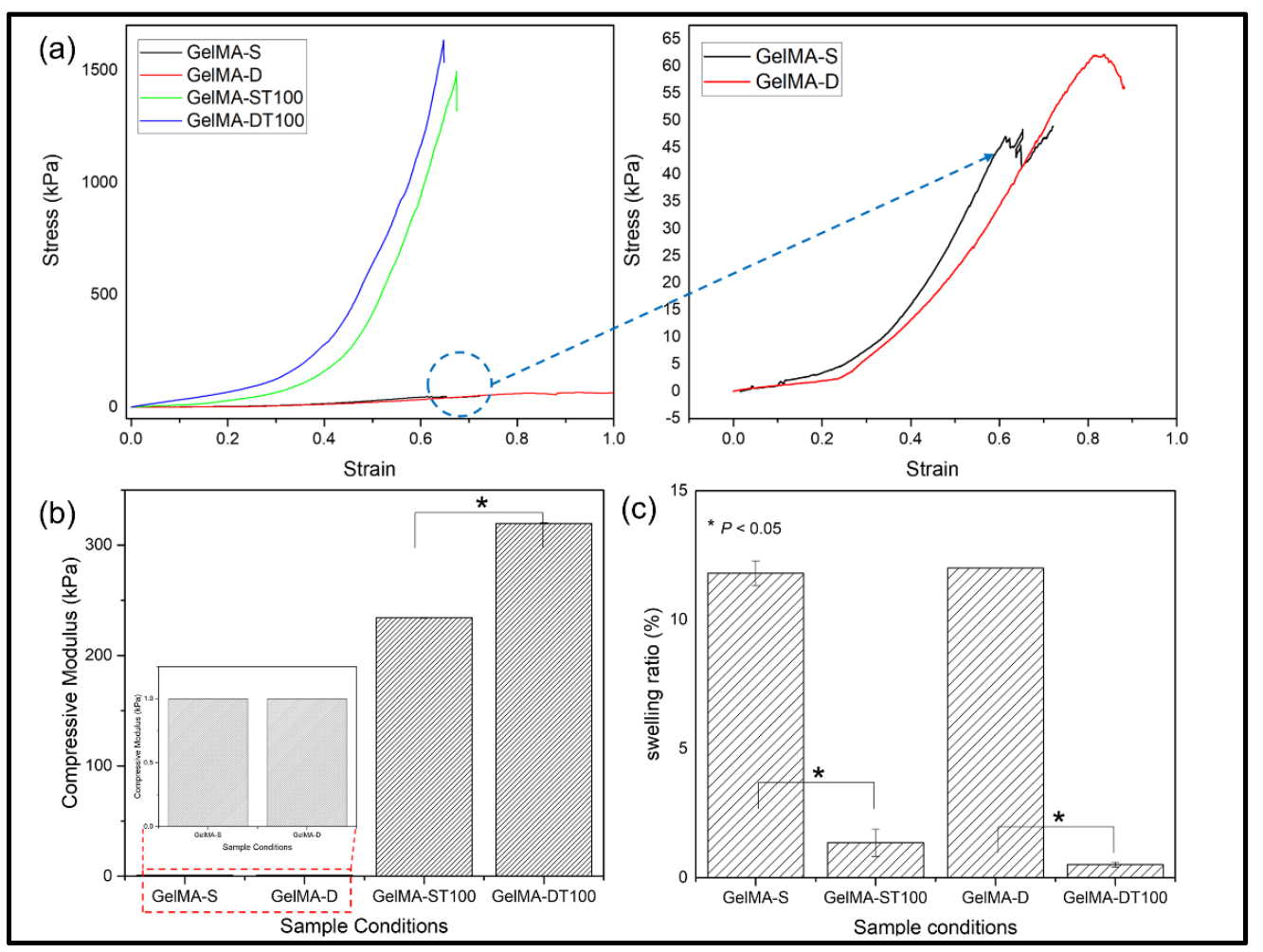

Figure 3. Mechanical properties of hydrogels (GelMA-S and GelMA-D) and after treated with the $100 w / v \%$ of TA. The compression deformation of all hydrogels was performed up to 95\%; (a) Stressstrain Curve for hydrogels, (b) Compressive Modulus through (0 20\% slope), (c) Swelling ratio of GelMA and GelMA-TA hydrogels. (Data are presented as mean $\pm \mathrm{SD}, n=3,{ }^{*} p<0.05$ ).

These results showed the same trend in the compressive stress (Figure $3 b$ ). The compressive stresses of each group were GelMA-S: $46 \mathrm{kPa}$; GelMA-D: $62 \mathrm{kPa}$; GelMAST100: $234 \mathrm{kPa}$; GelMA-DT100 showed $319 \mathrm{kPa}$, and it was confirmed that the improvement was about 1.3 times between the single crosslinked and double crosslinked groups. In addition, it was confirmed that there was a difference of about five times between the untreated group and the TA group. TA bound to the hydrogel strengthened the bond with the GelMA network through hydrogen bonding and hydrophobic force, and showed improvement in mechanical strength [49]. Mechanical properties can be affected by the water content of the hydrogel. Therefore, the GelMA-S, GelMA-D, GelMA-ST100, and GelMA-DT100 groups were immersed in PBS and then cultured in an incubator at $37^{\circ} \mathrm{C}$ for $24 \mathrm{~h}$. 
The smoothing behavior of the hydrogel was then characterized and shown in Figure 3c. The swelling behavior of GelMA-S and GelMA-D showed similar results, and no significant difference was observed. Similarly, no significant difference was observed in the swelling behavior of GelMA-ST100 and GelMA-DT100. However, a significant difference was observed when comparing the untreated group and the TA treated group. It could be confirmed that the TA-treated group was significantly restricted in swelling behavior compared to the untreated group. The low swelling behavior of the TA-treated group showed that the interaction of H-bonds between TA and GelMA could compress the structure of the hydrogel and limit water absorption [43]. As a result, it was confirmed that the low swelling ratio of the TA-treated hydrogel group indicates a higher mechanical strength.

\subsection{Cytocompatibility of Hydrogel}

We investigated the cellular compatibility of GelMA and GelMA-TA hydrogels. Proosteoblasts (MC3T3-E1) were cultured in flat 48-well plates with or without hydrogels. According to previously known research results, the differentiation of MC3T3-E1 cells showed better cell activity in soft materials than in hard materials [50]. The single and double crosslinked hydrogels used in the study have soft matrix properties, whereas the TA-treated GelMA hydrogels have more rigid matrix properties. Our experiments also showed similar results. Cell viability was confirmed using quantitative detection of cck8 at 1, 3, and 7 days after seeding (Figure 4). UV absorbance increased with increasing incubation time for days 1-3 due to cell proliferation, but there was no statistical difference in cell viability in all groups. However, on day 7 , there was a change between each group. GelMA-D showed better UV absorbance, and a significant difference occurred compared to other groups.

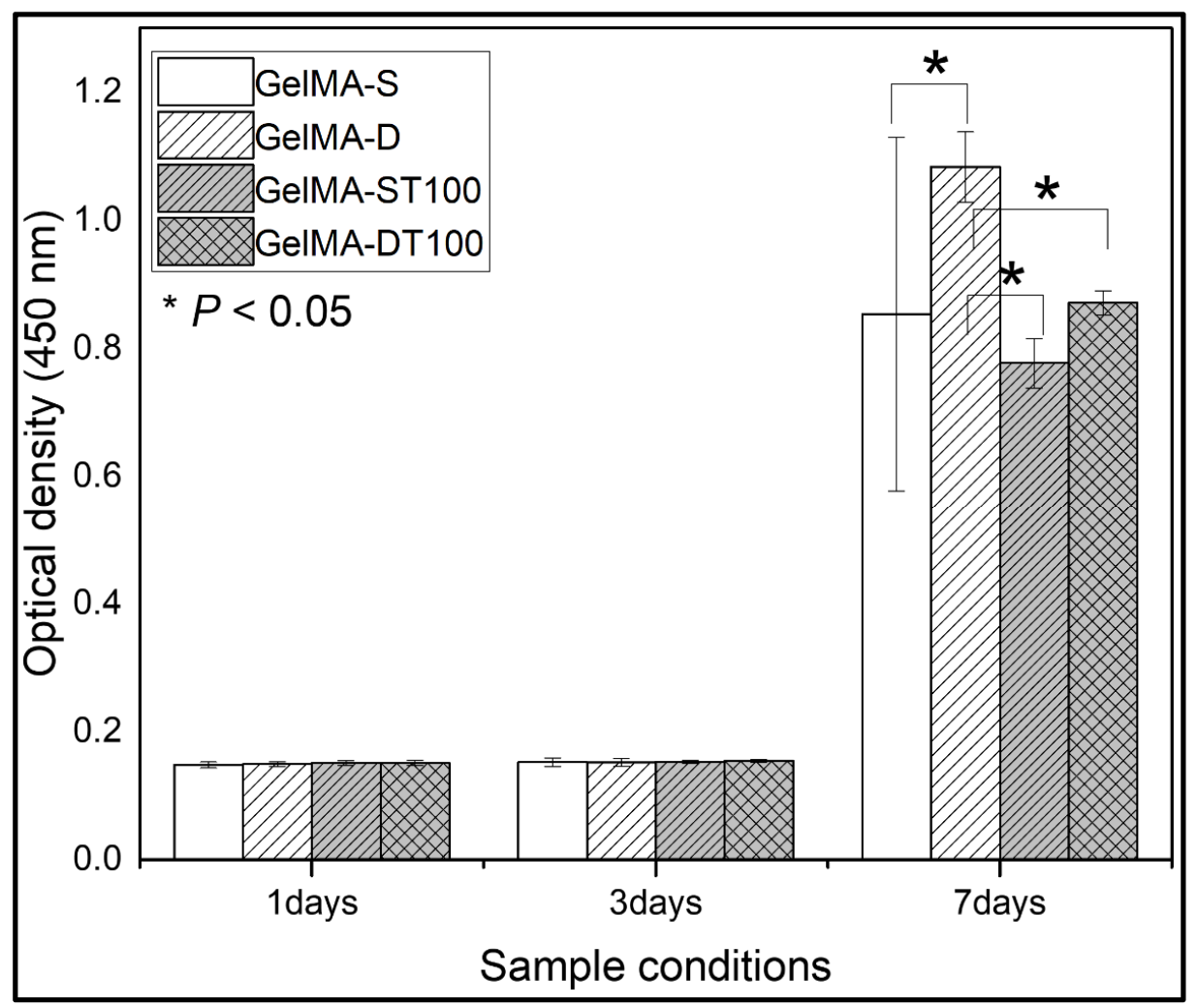

Figure 4. The proliferation of cells measured by CCK8 assays (WST) on days 1, 3, and 7. (Data are presented as mean $\left.\pm \mathrm{SD}, n=3,{ }^{*} p<0.05\right)$. 
The group treated with TA showed lower absorbance than the existing GelMA group, but there was no significant difference except for the GelMA-D group. These results also suggested that the hydrogel can be applied to in vivo use. A high concentration of TA treatment continuously released an excess of TA in the hydrogel, and it was confirmed that the released TA affects cell activity. Although the exact mechanism of the effect of TA on cellular activity is unknown, it may be due to the presence of galloyl groups in TA. A galloyl group of tannic acid interacts with proteins through hydrophobic bonding and electrostatic and hydrophobic interactions [51]. The electrostatic interaction induced by tannic acid at $7.4 \mathrm{pH}$ is relatively weaker than the hydrogen bonding and fails to stabilize proteins in their native form [52]. This interaction of tannic acid suggests the adhesion prevention of osteoblasts. Nevertheless, these results suggest that the hydrogel may be applicable for in vivo use.

\subsection{In Vivo Osteoinduction}

In vivo experiments were conducted with GelMA-S, GelMA-D, GelMA-ST100, and Gel-MA-DT100 groups. Osteoinduction and biodegradation were observed by implanting a hydrogel sample prepared in the form of a rod into the femur of a rat. After 4 and 8 weeks, mice were sacrificed and the results of new bone formation according to osteoinduction in the hydrogel are shown in Figure 5 through fluorescence images. Alizarin complexon (red) and calcein (green) staining was performed every 2 weeks to observe bone formation. After 4 weeks of sample transplantation, most of the staining intervals of alizarin red and calcein green were consistent, and there was no significant difference in the rate of new bone formation.

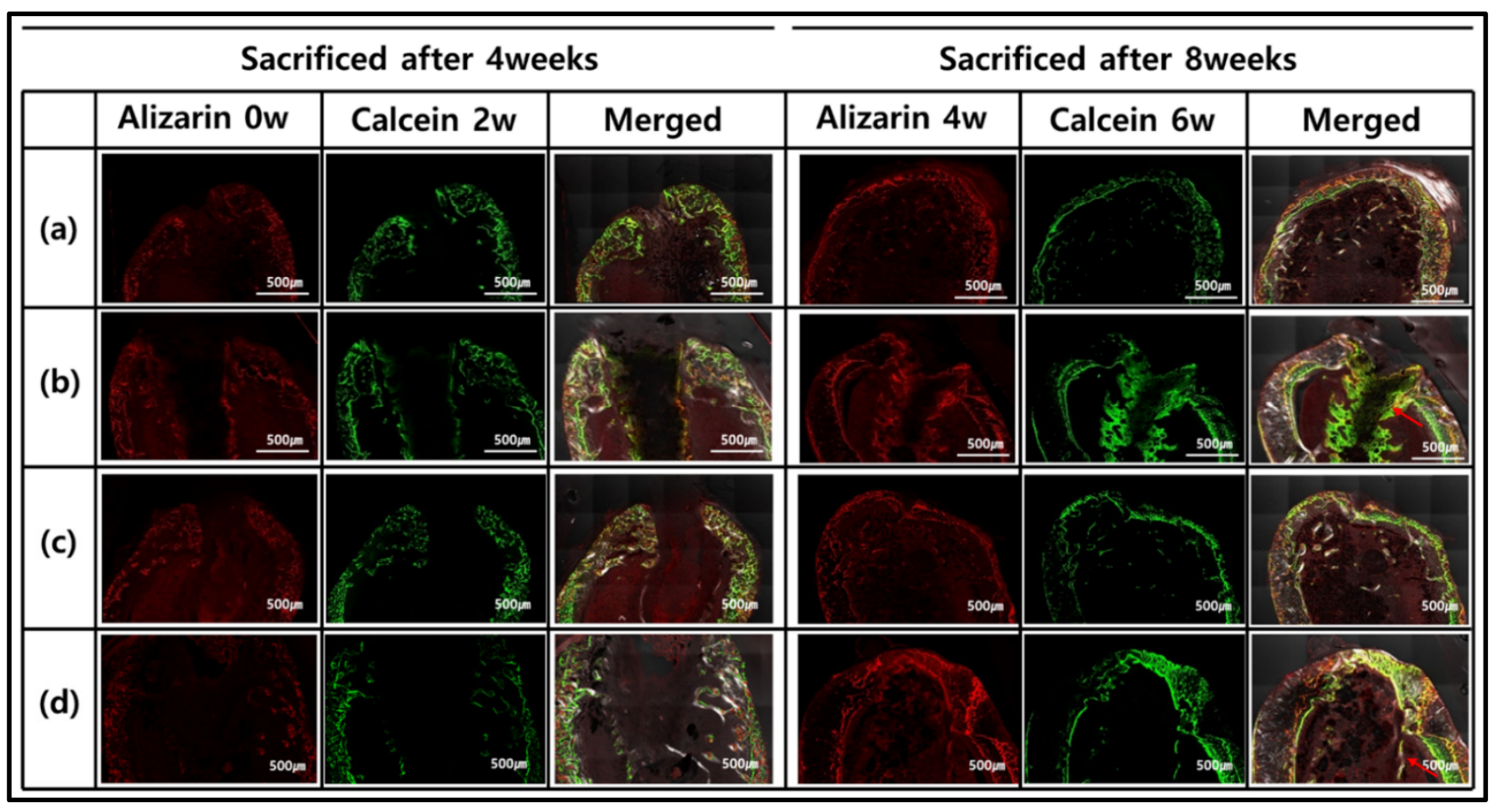

Figure 5. Fluorescence microscopy imaging after 4 and 8 weeks after implantation (red and green represent alizarin and calcein labeling, respectively): (a) GelMA-S, (b) GelMA-D, (c) GelMA-ST100, and (d) GelMA-DT100.

However, 8 weeks after implantation, different types were observed for each group. In GelMA-S and GelMA-ST100, the hydrogel morphology and osteoinductive reaction were not confirmed. On the other hand, it was confirmed that the formation of new bone and the concentration of calcein green increased in GelMA-D and GelMA-DT100. In particular, in GelMA-D, it was confirmed that many of these minerals were generated inside the hydrogel and maintained its shape. In the process of bone remodeling, bones 
are synthesized by osteoblasts, and small mineral crystals are deposited between collagen molecules. Over the next few days, these crystals fill the space occupied by water, resulting in bone mineralization [53]. For hydrogel samples, it is important to have adequate strength to retain their shape and the ability to absorb moisture. GelMA-D showed that many of the main minerals were settled in the hydrogel before the hydrogel was decomposed and replaced with the degradation (Figure 5b). However, in the hydrogel treated with TA, water absorption was inhibited, and it was confirmed that the main mineral was deposited only on the outside (Figure 5d). These morphologies tend to be consistent with Villanueva Osteochrome Staining (Figure 6).

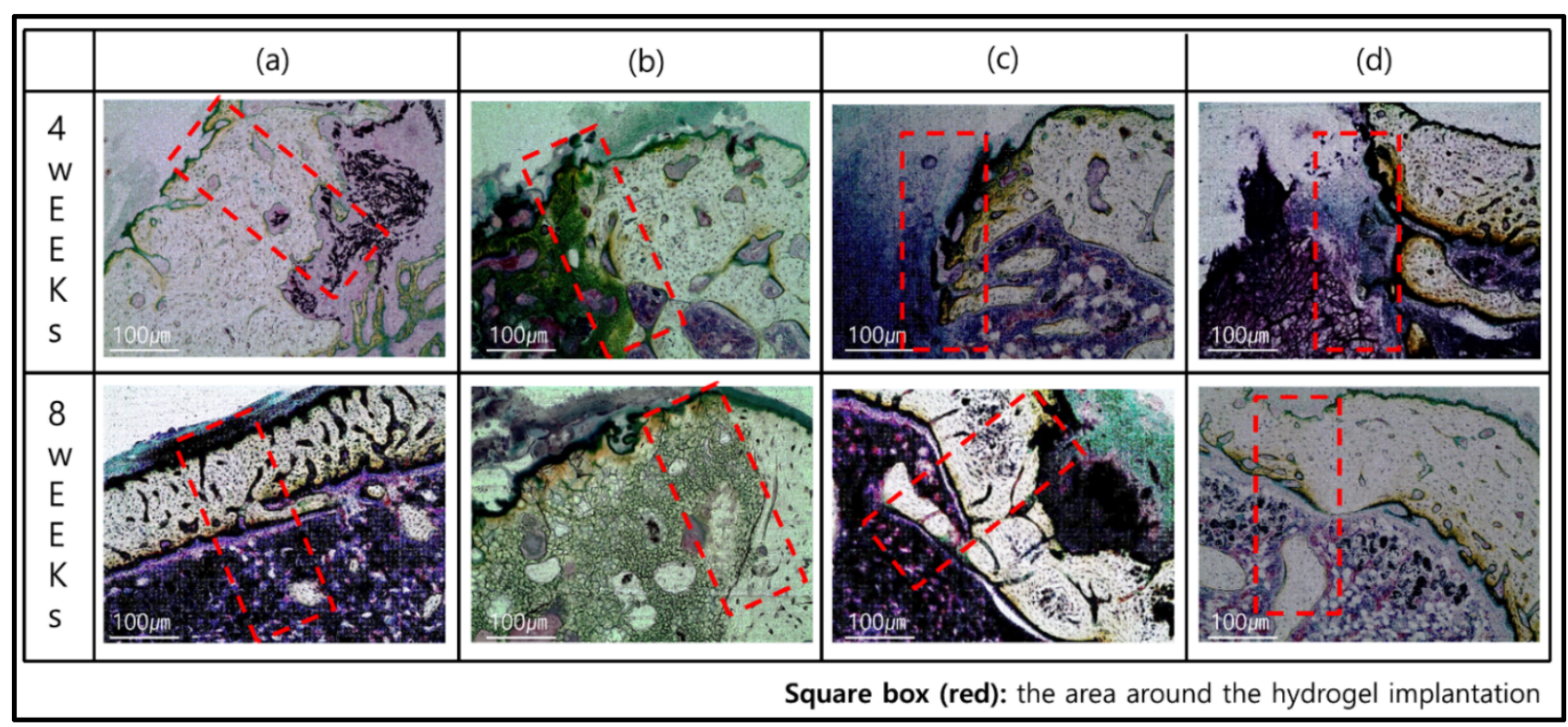

Figure 6. Histological images of Villanueva Osteochrome Staining for bone tissues after 4 and 8 weeks: (a) GelMA-S, (b) GelMA-D, (c) GelMA-ST100, and (d) GelMA-DT100.

GelMA-S did not adhere to the bone 4 weeks after transplantation, and it was confirmed that rapid degradation occurred (Figure 6a). On the other hand, in GelMA-D, osseointegration was successfully achieved 4 weeks after transplantation, and it was confirmed that the external bone mineral penetrated into the sample. In addition, it was observed that the bone mineral formed in the sample was gradually converted into the new bone after 8 weeks. It is judged that the induction reaction for bone remodeling occurred smoothly only in GelMA-D (Figure 6b). After 4 weeks of implantation of GelMA-ST100 and GelMA-DT100 treated with TA, the shape of the sample was maintained, but it did not adhere to the bone and there was no osteoinductive reaction (Figure $6 c, d$ ). The location where mineral crystals are deposited between collagen fibers, one of the components of bone, and where mineralization begins, is called the hole zone [52]. This region is the first to deposit calcium and phosphorus in the inter-molecular space created when collagen molecules move out of position when forming fibers [54].

GelMA-S can be exchanged smoothly in body fluids through its porous structure, but it has a disadvantage in that rapid degradation can occur because the reaction range by the enzyme is large. Double crosslinking solved these shortcomings. The improved strength provided a favorable environment for calcium and phosphorus deposition and new bone formation before being degraded by enzymes. On the other hand, TA treatment gave high mechanical strength but did not provide a suitable environment for the formation of new bone by osteoinduction. In addition, although the exact mechanism of TA has not been elucidated, it cannot be ruled out that the adhesion of progenitor cells is hindered by the nature of TA, which does not stabilize the natural protein in the environment of $\mathrm{pH}$ $7.4[55,56]$. 


\section{Conclusions}

In this study, it was evaluated whether a hydrogel produced by double crosslinking using GelMA, a natural polymer, was suitable for improving bone induction and showed better results when additionally combined with TA.

As a result, the double crosslinked group showed improved mechanical strength and better cell compatibility than the single crosslinked group. In addition, the single crosslinked group transplanted into the rat femur showed no osteoinduction response due to rapid degradation after 4 and 8 weeks, but the double crosslinked group showed bone mineral binding and osteoinduction. The hydrogel group treated with a high concentration of TA showed a significant improvement in mechanical strength through H-bonding. However, cell adhesion and proliferation were limited compared to the untreated group, and osteoinduction was not observed in the TA-treated single and double crosslinked groups.

In conclusion, it was found that double crosslinking is a suitable method for improving the strength and bone induction of hydrogels compared to single crosslinking. Conversely, it was confirmed that the binding of high-concentration TA significantly improved mechanical strength, but delayed bone remodeling by limiting water absorption. In future research, it is expected that the development of functional hydrogels that can be used for bone tissue engineering by supporting bioactive substances or drugs on the double crosslinked hydrogel is expected.

Author Contributions: Conceptualization, methodology, and writing-original draft preparation, J.-B.C.; writing-review and editing, S.-M.B.; validation and formal analysis, J.-E.P.; investigation and resources, T.-S.B.; project administration, Y.-K.K.; supervision and funding acquisition, M.-H.L. and Y.-S.J. All authors have read and agreed to the published version of the manuscript.

Funding: This research was funded by a National Research Foundation of Korea (NRF) grant funded by the Korea Government (MSIP) No. 2019R1C1C1003784.

Institutional Review Board Statement: The study was conducted according to the guidelines of the Declaration of Helsinki and approved by the Institutional Animal Care and Use Committee of the Chonbuk National University Laboratory Animal Center (CBNU 2020-094).

Informed Consent Statement: Not applicable.

Acknowledgments: This study has reconstructed the data of Ji-Bong Choi's doctoral dissertation submitted in 2021 (title: "Fabrication and characterization of biodegradable gelatin-methacrylate hydrogel for tissue engineering").

Conflicts of Interest: The authors declare no conflict of interest.

\section{References}

1. Giannoudis, P.V.; Dinopoulos, H.; Tsiridis, E. Bone substitutes: An update. Injury 2005, 36, S20-S27. [CrossRef] [PubMed]

2. Salgado, A.J.; Coutinho, O.P.; Reis, R.L. Bone tissue engineering: State of the art and future trends. Macromol. Biosci. 2004, 4, 743-765. [CrossRef] [PubMed]

3. Kim, S.; Von Recum, L. Endothelial stem cells and precursors for tissue engineering: Cell source, differentiation, selection, and application. Tissue Eng. Part B Rev. 2008, 14, 133-147. [CrossRef] [PubMed]

4. Annabi, N.; Tamayol, A.; Uquillas, J.A.; Akbari, M.; Bertassoni, L.E.; Cha, C.; Camci-Unal, G.; Dokmeci, M.R.; Peppas, N.A.; Khademhosseini, A. 25th anniversary article: Rational design and applications of hydrogels in regenerative medicine. Adv. Mater. 2014, 26, 85-124. [CrossRef]

5. Chen, Y.C.; Lin, R.Z.; Qi, H.; Yang, Y.; Bae, H.; Melero-Martin, J.M.; Khademhosseini, A. Functional human vascular network generated in photocrosslinkable gelatin methacrylate hydrogels. Adv. Funct. Mater. 2012, 22, 2027-2039. [CrossRef]

6. Nichol, J.W.; Koshy, S.T.; Bae, H.; Hwang, C.M.; Yamanlar, S.; Khademhosseini, A. Cell-laden microengineered gelatin methacrylate hydrogels. Biomaterials 2010, 31, 5536-5544. [CrossRef]

7. Lin, R.-Z.; Chen, Y.-C.; Moreno-Luna, R.; Khademhosseini, A.; Melero-Martin, J.M. Transdermal regulation of vascular network bioengineering using a photopolymerizable methacrylated gelatin hydrogel. Biomaterials 2013, 34, 6785-6796. [CrossRef]

8. Hosseini, V.; Ahadian, S.; Ostrovidov, S.; Camci-Unal, G.; Chen, S.; Kaji, H.; Ramalingam, M.; Khademhosseini, A. Engineered contractile skeletal muscle tissue on a microgrooved methacrylated gelatin substrate. Tissue Eng. Part A 2012, 18, $2453-2465$. [CrossRef] 
9. Park, C.; Vo, C.L.-N.; Kang, T.; Oh, E.; Lee, B.-J. New method and characterization of self-assembled gelatin-oleic nanoparticles using a desolvation method via carbodiimide/N-hydroxysuccinimide (EDC/NHS) reaction. Eur. J. Pharm. Biopharm. 2015, 89, 365-373. [CrossRef]

10. Yu, T.; Wang, W.; Nassiri, S.; Kwan, T.; Dang, C.; Liu, W.; Spiller, K.L. Temporal and spatial distribution of macrophage phenotype markers in the foreign body response to glutaraldehyde-crosslinked gelatin hydrogels. J. Biomater. Sci. Polym. Ed. 2016, 27, 721-742. [CrossRef]

11. Montemurro, F.; De Maria, C.; Orsi, G.; Ghezzi, L.; Tinè, M.R.; Vozzi, G. Genipin diffusion and reaction into a gelatin matrix for tissue engineering applications. J. Biomed. Mater. Res. Part B Appl. Biomater. 2017, 105, 473-480. [CrossRef]

12. Focaroli, S.; Teti, G.; Salvatore, V.; Durante, S.; Belmonte, M.M.; Giardino, R.; Mazzotti, A.; Bigi, A.; Falconi, M. Chondrogenic differentiation of human adipose mesenchimal stem cells: Influence of a biomimetic gelatin genipin crosslinked porous scaffold. Microsc. Res. Tech. 2014, 77, 928-934. [CrossRef]

13. Xie, X.; Tian, J.-K.; Lv, F.-Q.; Wu, R.; Tang, W.-B.; Luo, Y.-K.; Huang, Y.-Q.; Tang, J. A novel hemostatic sealant composed of gelatin, transglutaminase and thrombin effectively controls liver trauma-induced bleeding in dogs. Acta Pharmacol. Sin. 2013, 34, 983-988 [CrossRef]

14. Van Den Bulcke, A.I.; Bogdanov, B.; De Rooze, N.; Schacht, E.H.; Cornelissen, M.; Berghmans, H. Structural and rheological properties of methacrylamide modified gelatin hydrogels. Biomacromolecules 2000, 1, 31-38. [CrossRef]

15. Benton, J.A.; De Forest, C.A.; Vivekanandan, V.; Anseth, K.S. Photocrosslinking of gelatin macromers to synthesize porous hydrogels that promote valvular interstitial cell function. Tissue Eng. Part A 2009, 15, 3221-3230. [CrossRef]

16. Schuurman, W.; Levett, P.A.; Pot, M.W.; van Weeren, P.R.; Dhert, W.J.; Hutmacher, D.W.; Melchels, F.P.; Klein, T.J.; Malda, J. Gelatin-methacrylamide hydrogels as potential biomaterials for fabrication of tissue-engineered cartilage constructs. Macromol. Biosci. 2013, 13, 551-561. [CrossRef]

17. Liu, W.; Heinrich, M.A.; Zhou, Y.; Akpek, A.; Hu, N.; Liu, X.; Guan, X.; Zhong, Z.; Jin, X.; Khademhosseini, A. Extrusion bioprinting of shear-thinning gelatin methacryloyl bioinks. Adv. Healthc. Mater. 2017, 6, 1601451. [CrossRef]

18. Zhao, X.; Sun, X.; Yildirimer, L.; Lang, Q.; Lin, Z.Y.W.; Zheng, R.; Zhang, Y.; Cui, W.; Annabi, N.; Khademhosseini, A. Cell infiltrative hydrogel fibrous scaffolds for accelerated wound healing. Acta Biomater. 2017, 49, 66-77. [CrossRef]

19. Hu, J.; Hou, Y.; Park, H.; Choi, B.; Hou, S.; Chung, A.; Lee, M. Visible light crosslinkable chitosan hydrogels for tissue engineering. Acta Biomater. 2012, 8, 1730-1738. [CrossRef]

20. Lee, B.H.; Lum, N.; Seow, L.Y.; Lim, P.Q.; Tan, L.P. Synthesis and characterization of types A and B gelatin methacryloyl for bioink applications. Materials 2016, 9, 797. [CrossRef]

21. Noshadi, I.; Hong, S.; Sullivan, K.E.; Sani, E.S.; Portillo-Lara, R.; Tamayol, A.; Shin, S.R.; Gao, A.E.; Stoppel, W.L.; Black, L.D., III. In vitro and in vivo analysis of visible light crosslinkable gelatin methacryloyl (GelMA) hydrogels. Biomater. Sci. 2017, 5, 2093-2105. [CrossRef]

22. Fedorovich, N.E.; Oudshoorn, M.H.; van Geemen, D.; Hennink, W.E.; Alblas, J.; Dhert, W.J. The effect of photopolymerization on stem cells embedded in hydrogels. Biomaterials 2009, 30, 344-353. [CrossRef]

23. Kappes, U.P.; Luo, D.; Potter, M.; Schulmeister, K.; Rünger, T.M. Short-and long-wave UV light (UVB and UVA) induce similar mutations in human skin cells. J. Investig. Dermatol. 2006, 126, 667-675. [CrossRef]

24. Jones, C.A.; Huberman, E.; Cunningham, M.L.; Peak, M.J. Mutagenesis and cytotoxicity in human epithelial cells by far-and near-ultraviolet radiations: Action spectra. Radiat. Res. 1987, 110, 244-254. [CrossRef]

25. Kielbassa, C.; Roza, L.; Epe, B. Wavelength dependence of oxidative DNA damage induced by UV and visible light. Carcinogenesis 1997, 18, 811-816. [CrossRef]

26. Elisseeff, J.; Anseth, K.; Sims, D.; McIntosh, W.; Randolph, M.; Langer, R. Transdermal photopolymerization for minimally invasive implantation. Proc. Natl. Acad. Sci. USA 1999, 96, 3104-3107. [CrossRef]

27. Sadat-Shojai, M.; Khorasani, M.-T.; Jamshidi, A. 3-dimensional cell-laden nano-hydroxyapatite/protein hydrogels for bone regeneration applications. Mater. Sci. Eng. C 2015, 49, 835-843. [CrossRef]

28. Zuo, Y.; Liu, X.; Wei, D.; Sun, J.; Xiao, W.; Zhao, H.; Guo, L.; Wei, Q.; Fan, H.; Zhang, X. Photo-cross-linkable methacrylated gelatin and hydroxyapatite hybrid hydrogel for modularly engineering biomimetic osteon. ACS Appl. Mater. Interfaces 2015, 7, 10386-10394. [CrossRef]

29. Annabi, N.; Rana, D.; Sani, E.S.; Portillo-Lara, R.; Gifford, J.L.; Fares, M.M.; Mithieux, S.M.; Weiss, A.S. Engineering a sprayable and elastic hydrogel adhesive with antimicrobial properties for wound healing. Biomaterials 2017, 139, 229-243. [CrossRef]

30. Rothrauff, B.B.; Yang, G.; Tuan, R.S. Tissue-specific bioactivity of soluble tendon-derived and cartilage-derived extracellular matrices on adult mesenchymal stem cells. Stem Cell Res. Ther. 2017, 8, 133. [CrossRef]

31. Chen, X.; Bai, S.; Li, B.; Liu, H.; Wu, G.; Liu, S.; Zhao, Y. Fabrication of gelatin methacrylate/nanohydroxyapatite microgel arrays for periodontal tissue regeneration. Int. J. Nanomed. 2016, 11, 4707. [CrossRef]

32. Bartnikowski, M.; Akkineni, A.R.; Gelinsky, M.; Woodruff, M.A.; Klein, T.J. A hydrogel model incorporating 3D-plotted hydroxyapatite for osteochondral tissue engineering. Materials 2016, 9, 285. [CrossRef] [PubMed]

33. Eslami, M.; Vrana, N.E.; Zorlutuna, P.; Sant, S.; Jung, S.; Masoumi, N.; Khavari-Nejad, R.A.; Javadi, G.; Khademhosseini, A. Fiber-reinforced hydrogel scaffolds for heart valve tissue engineering. J. Biomater. Appl. 2014, 29, 399-410. [CrossRef] [PubMed] 
34. Ramón-Azcón, J.; Ahadian, S.; Estili, M.; Liang, X.; Ostrovidov, S.; Kaji, H.; Shiku, H.; Ramalingam, M.; Nakajima, K.; Sakka, Y. Dielectrophoretically aligned carbon nanotubes to control electrical and mechanical properties of hydrogels to fabricate contractile muscle myofibers. Adv. Mater. 2013, 25, 4028-4034. [CrossRef]

35. Cha, C.; Shin, S.R.; Gao, X.; Annabi, N.; Dokmeci, M.R.; Tang, X.; Khademhosseini, A. Controlling mechanical properties of cell-laden hydrogels by covalent incorporation of graphene oxide. Small 2014, 10, 514-523. [CrossRef]

36. Zawko, S.A.; Suri, S.; Truong, Q.; Schmidt, C.E. Photopatterned anisotropic swelling of dual-crosslinked hyaluronic acid hydrogels. Acta Biomater. 2009, 5, 14-22. [CrossRef]

37. Rizwan, M.; Peh, G.S.; Ang, H.-P.; Lwin, N.C.; Adnan, K.; Mehta, J.S.; Tan, W.S.; Yim, E.K. Sequentially-crosslinked bioactive hydrogels as nano-patterned substrates with customizable stiffness and degradation for corneal tissue engineering applications. Biomaterials 2017, 120, 139-154. [CrossRef]

38. Zhou, M.; Lee, B.H.; Tan, L.P. A dual crosslinking strategy to tailor rheological properties of gelatin methacryloyl. Int. J. Bioprint. 2017. [CrossRef]

39. Sahiner, N.; Sagbas, S.; Sahiner, M.; Silan, C.; Aktas, N.; Turk, M. Biocompatible and biodegradable poly (Tannic Acid) hydrogel with antimicrobial and antioxidant properties. Int. J. Biol. Macromol. 2016, 82, 150-159. [CrossRef]

40. Le Bourvellec, C.; Renard, C. Interactions between polyphenols and macromolecules: Quantification methods and mechanisms. Crit. Rev. Food Sci. Nutr. 2012, 52, 213-248. [CrossRef]

41. Chen, Y.-N.; Peng, L.; Liu, T.; Wang, Y.; Shi, S.; Wang, H. Poly (vinyl alcohol)-tannic acid hydrogels with excellent mechanical properties and shape memory behaviors. ACS Appl. Mater. Interfaces 2016, 8, 27199-27206. [CrossRef]

42. Shin, M.; Ryu, J.H.; Park, J.P.; Kim, K.; Yang, J.W.; Lee, H. DNA/tannic acid hybrid gel exhibiting biodegradability, extensibility, tissue adhesiveness, and hemostatic ability. Adv. Funct. Mater. 2015, 25, 1270-1278. [CrossRef]

43. Choi, J.-B.; Kim, Y.-K.; Byeon, S.-M.; Park, J.-E.; Bae, T.-S.; Jang, Y.-S.; Lee, M.-H. Fabrication and characterization of biodegradable gelatin methacrylate/biphasic calcium phosphate composite hydrogel for bone tissue engineering. Nanomaterials 2021, 11, 617. [CrossRef]

44. Fan, H.; Wang, L.; Feng, X.; Bu, Y.; Wu, D.; Jin, Z. Supramolecular hydrogel formation based on tannic acid. Macromolecules 2017, 50, 666-676. [CrossRef]

45. Liu, B.; Wang, Y.; Miao, Y.; Zhang, X.; Fan, Z.; Singh, G.; Zhang, X.; Xu, K.; Li, B.; Hu, Z. Hydrogen bonds autonomously powered gelatin methacrylate hydrogels with super-elasticity, self-heal and underwater self-adhesion for sutureless skin and stomach surgery and E-skin. Biomaterials 2018, 171, 83-96. [CrossRef] [PubMed]

46. Zhao, X.; Lang, Q.; Yildirimer, L.; Lin, Z.Y.; Cui, W.; Annabi, N.; Ng, K.W.; Dokmeci, M.R.; Ghaemmaghami, A.M.; Khademhosseini, A. Photocrosslinkable gelatin hydrogel for epidermal tissue engineering. Adv. Healthc. Mater. 2016, 5, 108-118. [CrossRef]

47. Kozlovskaya, V.; Kharlampieva, E.; Drachuk, I.; Cheng, D.; Tsukruk, V.V. Responsive microcapsule reactors based on hydrogenbonded tannic acid layer-by-layer assemblies. Soft Matter 2010, 6, 3596-3608. [CrossRef]

48. Mao, A.S.; Shin, J.-W.; Mooney, D.J. Effects of substrate stiffness and cell-cell contact on mesenchymal stem cell differentiation. Biomaterials 2016, 98, 184-191. [CrossRef]

49. Coleman, M.M.; Lee, K.H.; Skrovanek, D.J.; Painter, P.C. Hydrogen bonding in polymers. 4. Infrared temperature studies of a simple polyurethane. Macromolecules 1986, 19, 2149-2157. [CrossRef]

50. Ma, M.; Zhong, Y.; Jiang, X. Thermosensitive and $\mathrm{pH}$-responsive tannin-containing hydroxypropyl chitin hydrogel with longlasting antibacterial activity for wound healing. Carbohydr. Polym. 2020. [CrossRef]

51. Topkaya, S.N. Gelatin methacrylate (GelMA) mediated electrochemical DNA biosensor for DNA hybridization. Biosens. Bioelectron. 2015, 64, 456-461. [CrossRef] [PubMed]

52. Muhoza, B.; Xia, S.; Zhang, X. Gelatin and high methyl pectin coacervates crosslinked with tannic acid: The characterization, rheological properties, and application for peppermint oil microencapsulation. Food Hydrocoll. 2019, 97, 105174. [CrossRef]

53. Glimcher, M.J. Mechanism of calcification: Role of collagen fibrils and collagen-phosphoprotein complexes in vitro and in vivo. Anat. Rec. 1989, 224, 139-153. [CrossRef] [PubMed]

54. Shin, C.S.; Cho, J.Y. Bone remodeling and mineralization. J. Korean Endocrinol. Soc. 2005, 20, doi. [CrossRef]

55. Yu, X.; Biedrzycki, A.H.; Khalil, A.S.; Hess, D.; Umhoefer, J.M.; Markel, M.D.; Murphy, W.L. Nanostructured mineral coatings stabilize proteins for therapeutic delivery. Adv. Mater. 2017, 29, 1701255. [CrossRef]

56. Minteer, S.D. Enzyme Stabilization and Immobilization; Springer: Berlin/Heidelberg, Germany, 2017; pp. 94-104. 